\title{
Analysis of Decentralized Integral Controllability for Nonlinear Systems
}

\author{
Steven W. $\mathrm{Su}^{a}$, Jie $\mathrm{Bao}^{a *}$ and Peter L. Lee ${ }^{b}$ \\ ${ }^{a}$ School of Chemical Engineering \& Industrial Chemistry \\ The University of New South Wales, \\ UNSW, Sydney, NSW 2052, Australia \\ ${ }^{b}$ Division of Engineering, Science \& Computing \\ Curtin University of Technology \\ GPO Box U1987, Perth, WA 6845, Australia
}

\begin{abstract}
This paper extends the concept of Decentralized Integral Controllability to nonlinear process, and presents a steady state sufficient condition by using the singular perturbation theory. A numerical method is provided to facilitate the analysis of the proposed condition and is illustrated using an example of a dual tank level control system.
\end{abstract}

Keywords: Decentralized Integral Controllability, Nonlinear Processes, Process Control 


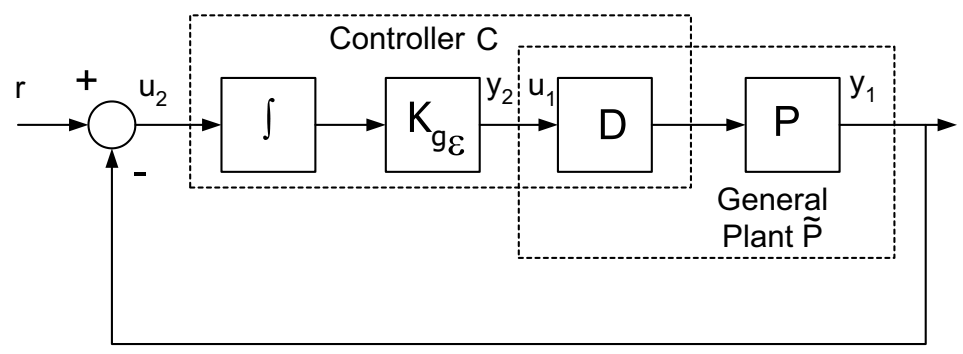

Figure 1: Decentralized Integral Controllability

\section{Introduction}

Decentralized control is the most widely used strategy in industrial process control due to its design and hardware simplicity, fault tolerance, and operator acceptance. For decentralized designs, an important issue is Decentralized Integral Controllability (DIC). Introduced by Skogestad and Morari (Skogestad \& Morari 1988), DIC analysis determines whether a multivariable plant can be stabilized by multi-loop controllers, whether the controller can have integral action to achieve offset free control, and whether the closed-loop system will remain stable when any subset of loops is detuned or taken out of service. DIC is a property of a given plant in combination with some pre-specified control structure (such as choices of manipulated and controlled variables and pairings). A comprehensive literature for DIC conditions for linear processes have been developed. As the necessary and sufficient conditions (Le, Nwokah \& Frazho 1991, Nwokah, Frazho \& Le 1993) are often very difficult to use for processes of large dimensions, convenient DIC conditions are either necessary or sufficient. While necessary conditions (Skogestad \& Morari 1988, Yu \& Fan 1990, Campo \& Morari 1994, Lee \& Edgar 2002) are often used to screen out unsuitable pairings, the sufficient conditions (Skogestad \& Morari 1988, Morari \& Zafiriou 1989, Campo \& Morari 1994, Bao, McLellan \& Forbes 2002) are useful to deduce whether a process with a particular control structure possesses the DIC property.

This paper extends the concept of DIC to nonlinear processes and provides a new sufficient nonlinear DIC condition. As most chemical processes are nonlinear, the usual practice is to perform controllability analysis based on linearized models (Nikolaou \& Misra 2003). This approach, however, could produce misleading results as they only indicate the controllability within a (maybe arbitrarily) small vicinity around the operating point (Luyben, Tyrus \& Luyben 1999, Vinson \& Georgakis 1999). It is clear that nonlinear DIC conditions are useful in providing both theoretical insights of nonlinear decentralized control and analysis tools for control practice. This paper is organized as follows. The concept of nonlinear DIC is defined in Section 2. A new sufficient DIC condition is proposed based on the singular perturbation theory in Section 3. A numerical method to implement the proposed DIC analysis is presented using an illustrative example in Section 4.

\section{Decentralized integral controllability for nonlinear systems}

The concept of Decentralized Integral Controllability for nonlinear systems is defined as an extension of linear DIC (Skogestad \& Morari 1988). It indicates whether a nonlinear multivariable process can be stabilized (the precise type of stabilization will be discussed later) by multi-loop linear or nonlinear controllers with integral 
action to ensure zero steady-state error and whether the closed-loop stability is guaranteed when any subset of loop is arbitrarily detuned. As shown in Figure 1, the $m$-loop decentralized nonlinear controller $C$ with integral action can be decomposed into three components: the stable diagonal nonlinear controller $D$, the gain matrix $K_{g}=\operatorname{diag}\left\{k_{i}\right\}\left(k_{i}>0, i=1,2, \cdots, m\right)$ and the integrator. The problem of DIC can be interpreted as whether the closed loop system is stable and remains stable when $K_{g}$ is reduced to $K_{g \varepsilon}=\operatorname{diag}\left\{k_{i} \varepsilon_{i}\right\}$, $0 \leq \varepsilon_{i} \leq 1, i=1, \cdots, m$. This is similar to the interpretation for linear DIC (Bao et al. 2002). However, different from linear DIC analysis, the stability for nonlinear systems normally has to be analyzed for a particular equilibrium point. As shown in Figure 1, we restrict our attention to the "square" dynamical process modeled by the following equations with an input vector $u \in \mathcal{R}^{m}$ and an output vector $y \in \mathcal{R}^{m}$ :

$$
P \begin{cases}\dot{x}=f(x, u) \quad & x \in X \subset \mathcal{R}^{n}, u \in \mathcal{U} \subset \mathcal{R}^{m} \\ y=g(x, u), & y \in y \subset \mathcal{R}^{m},\end{cases}
$$

It is assumed that the state $x(t)$ is uniquely determined by its initial value $x(0)$ and the input function $u(t)$. Another assumption made for convenience is that the system (1) has equilibrium at origin, that is, $f(0,0)=0$, and $g(0,0)=0$. If the equilibrium $x_{e}$ is not at origin, a translation is needed by redefining the state $x$ as $x-x_{e}$. The nonlinear DIC is defined as follows:

Definition 1 (Decentralized Integral Controllability for nonlinear processes)

Consider the closed loop system depicted in Figure 1.

(i) For the nonlinear process $P$ described by equation (1), if there exists a decentralized integral controller $C$, such that the unforced closed loop system $(r=0)$ is Globally Asymptotically Stable (GAS) (Sepulchre, Jankovic 8 Kokotovic 1996) for the equilibrium $x=0$ and such that the Globally Asymptotically Stability is maintained if each individual loop is detuned independently by a factor $\varepsilon_{i}\left(0 \leq \varepsilon_{i} \leq 1, i=1, \cdots, m\right)$, then the nonlinear process $P$ is said to be Decentralized Integral Controllable(DIC) for the equilibrium $x=0$.

(ii) If the closed loop system is Asymptotically Stable $(A S)$ near the region of the equilibrium $x=0$, then the nonlinear process $P$ is said to be locally Decentralized Integral Controllable around the equilibrium $x=0$.

From the above definition, it is obvious that the process $P$ has to be Globally Asymptotically Stable around the equilibrium $x=0$ to be DIC. System $D$ is generally nonlinear. If a linear diagonal system $D$ can be found such that the closed-loop stability in the above DIC definition can be achieved, then this process is Decentralized Integral Controllable by using a linear controller. This is a desirable property as linear control systems usually require substantially lower design, implementation, and maintenance demands than nonlinear control systems (Nikolaou \& Misra 2003). The definition of local DIC is only concerned with the neighborhood of the equilibrium point. Normally, it can be assessed by applying existing linear DIC conditions to a linearized model around a particular equilibrium point.

\section{Steady-state DIC conditions for nonlinear processes}

In this section, a sufficient DIC condition is derived for nonlinear systems. By using the singular perturbation theory, it is found that, like the sufficient DIC conditions for linear processes (Yu \& Fan 1990, Campo \& 
Morari 1994, Bao et al. 2002, Lee \& Edgar 2002), the Decentralized Integral Controllability of a nonlinear process can be determined based on its input-output relationship at steady-state.

To analyze nonlinear DIC in the framework of standard singular perturbation problem, the system in Figure 1 is re-arranged as shown in Figure 2, where $K_{g \varepsilon}$ in Figure 1 is replaced by $\eta K_{g \varepsilon}^{\prime}$ where $\eta$ is a small positive real scalar. The elements of diagonal matrix $K_{g}$ are adjusted such that $K_{g}$ is positive definite. This can be performed by multiplying the diagonal elements of matrix $K_{g}$ by +1 or -1 . The sign adjustment is absorbed into the diagonal controller $D$.

In Figure 2, we suppose that the state equation of the general process $\tilde{P}$ (which is the serial connection of process $P$ and the diagonal controller $D$ ) is modeled as below (with the same assumptions for equation (1) of process $P$ ).

$$
\tilde{P}:\left\{\begin{array}{l}
\dot{x}=f\left(x, u_{1}\right) \\
y_{1}=g\left(x, u_{1}\right) .
\end{array}\right.
$$

The state equation for the linear integral controller is expressed as:

$$
C_{l}:\left\{\begin{array}{l}
\dot{\xi}=\eta K_{g \varepsilon}^{\prime} u_{2} \\
y_{2}=\xi
\end{array}\right.
$$

The following definition of Globally Exponential Stability (GES) is useful in developing the DIC condition:

Definition 2 (Globally Exponential Stability)

A system is globally exponentially stable (GES) if and only if there exists a Lyapunov function $U(x)$ such that

$$
\rho_{1}|x|^{2} \leq U(x) \leq \rho_{2}|x|^{2}
$$

and with zero input

$$
\frac{d}{d t} U(x(t)) \leq-\rho_{3}|x|^{2}
$$

Where $\rho_{i}>0, i=1,2,3$ are suitable scalar constants. If these conditions hold, it follows that there exists some constant $\rho \geq 0$ such that with $x(0)=x_{0},|x(t)| \leq \rho\left|x_{0}\right| e^{-\rho_{3} t / 2}$ for all $t \geq 0$.

Locally Exponential Stability (LES) implies that this definition is valid at least for $x$ in a neighborhood of $x=0$.

Theorem 3 (steady-state DIC conditions for nonlinear process)

Consider the closed loop system in Figure 2, and assume that the general process $\tilde{P}$ and the linear part of the controller $C_{l}$ are described by equation (2) and (3) respectively. If the following assumptions are satisfied:

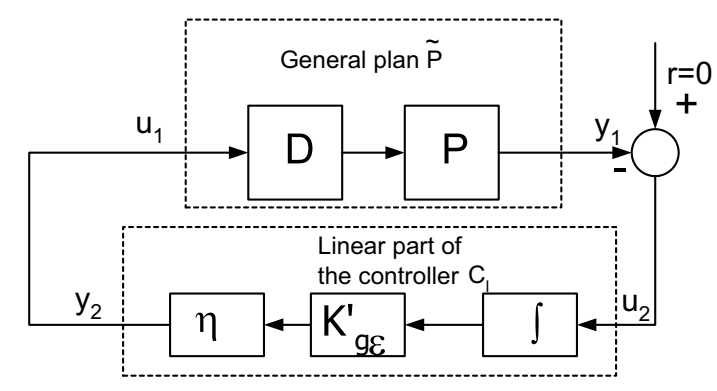

Figure 2: DIC analysis for nonlinear process by using singular perturbation analysis 
(i) The equation $0=f\left(x, u_{1}\right)$ obtained by setting $\eta=0$ in equation (2) implicitly defines a unique $C^{2}$ function $x=h\left(u_{1}\right)$ for $u_{1} \in \mathcal{U}_{1} \subset \mathcal{R}^{m}$.

(ii) For any fixed $u_{1} \in \mathcal{U}_{1} \subset \mathcal{R}^{m}$, the equilibrium $x_{e}=h\left(u_{1}\right)$ of the system $\dot{x}=f\left(x, u_{1}\right)$ is Globally Asymptotically Stable (GAS) and Locally Exponentially Stable (LES).

(iii) The steady-state input output function $g\left(h\left(u_{1}\right), u_{1}\right)$ of the general process $\tilde{P}$ satisfies the following requirements: $u_{1}^{T} g\left(h\left(u_{1}\right), u_{1}\right)>0$ (when $u_{1} \neq 0$ and $\left.u_{1} \in \mathcal{U}_{1}\right)$ and $u_{1}^{T} g\left(h\left(u_{1}\right), u_{1}\right) \geq \rho\left|u_{1}\right|^{2}$ (for some scalar $\rho>0$ ) for $u_{1}$ in a neighborhood of $u_{1}=0$.

Then there exists $\eta>0$, such that the equilibrium is GAS. That is, if a diagonal MIMO controller D can be found such that the serial connection of the nonlinear controller $D$ and the nonlinear process $P$ can satisfy Conditions i), ii) and iii), then the nonlinear MIMO process is DIC for the equilibrium.

Proof The proof is in Appendix A.

The conditions associated with Theorem 3 are all for the general process $\tilde{P}$ (which is the serial connection of process $P$ and the diagonal controller $D$ ) rather than the nonlinear plant $P$. To verify the above DIC conditions, a diagonal nonlinear controller $D$ needs to be constructed. Searching for a linear diagonal system $D$ is usually attempted first as it is much easier to construct than a nonlinear system. In this case, the overall controller will be linear.

If we denote $G_{0}$ as the steady-state gain matrix of the linearized model of $P$ around the equilibrium, and simply choose $D$ as a real diagonal matrix, then, Condition iii) in Theorem 3 is reduced to the existence of a real diagonal matrix $D_{l}=\operatorname{diag}\left\{d_{1}, d_{2}, \cdots, d_{m}\right\}\left(d_{i} \neq 0, i \in\{1,2, \cdots, m\}\right)$ such that

$$
G_{0} D_{l}+D_{l} G_{0}^{T}>0
$$

which is a sufficient condition for local DIC (See Definition 1). This is similar to the steady-state gain matrix condition for linear systems (Theorem 1 in (Bao et al. 2002) or Lemma 5 in (Campo \& Morari 1994)). If inequality (4) is not satisfied, then the global conditions (or in the region of interest) of Theorem 3 cannot hold. As the local DIC condition is much easier to test, it can be used to facilitate the analysis of global DIC.

The necessary conditions for linear processes (e.g.,(Yu \& Fan 1990, Campo \& Morari 1994, Lee \& Edgar 2002)) can be used as necessary conditions for nonlinear DIC to eliminate unworkable designs or parings. A mild necessary condition of process $P$ being DIC for both local and global around the equilibrium can be drawn from Theorem 8 in (Campo \& Morari 1994). The easy to use necessary conditions were given as Theorem 4 and 5 in (Lee \& Edgar 2002), and Theorem 4 in (Yu \& Fan 1990). It can be proved that the above linear necessary DIC conditions are local necessary nonlinear DIC conditions by using singular perturbation analysis (Kokotovic, Khalil \& Reilly 1986).

\section{Numerical method for DIC Analysis}

For any physical processes, DIC only needs to be assessed for the operating region of interest instead of a "theoretical" global space. Similar to other nonlinear analysis, test of the DIC conditions of Theorem 3 analytically is often difficult and sometimes even impossible. In this section, a numerical method is introduced 
to assess the DIC for a given nonlinear process $P: u \rightarrow y$, within the operating region of $u \in \mathcal{U} \subset \mathcal{R}^{m}$, $y \in y \subset \mathcal{R}^{m}$, with respect to the steady-state equilibrium point $\left(u_{e}, y_{e}\right)$.

Procedure 4 (Numerical analysis of nonlinear DIC)

Step 1. Check the stability (GAS and LES) of the nonlinear process P (Condition (ii) of Theorem 3). If process $P$ is unstable, it is not DIC.

Step 2. Linearize the nonlinear process $P$ around the equilibrium point, and check whether the steadystate gain matrix $G(0)$ of the linearized model satisfies the necessary conditions for linear systems (e.g., the necessary conditions in (Morari \& Zafiriou 1989, Yu 85 Fan 1990, Campo 6 Morari 1994, Lee E Edgar 2002)). If any of these necessary conditions cannot be satisfied, then process $P$ does not possess the DIC property. Otherwise go to the next step.

Step 3. Test the sufficient condition for linear DIC in equation (4) using the linearized model around the equilibrium point. This can be undertaken by using the numerical method based on Semi-Definite Programming given in (Bao et al. 2002) to find a real diagonal constant matrix $D_{l}$ that satisfies inequality (4). If the sufficient DIC condition for linear systems is not satisfied, DIC of the nonlinear process P cannot be concluded. Otherwise, proceed to the next step.

Step 4. Check whether Condition (i) of Theorem 3 is satisfied by the nonlinear model (2) of the general process $\tilde{P}: u_{1} \rightarrow y\left(u_{1} \in \mathcal{U}_{1} \subset \mathcal{R}^{m}, y \in y \subset \mathcal{R}^{m}\right)$, where $\tilde{P}$ is process $P$ serially connected with the diagonal system $D . \mathcal{U}_{1}$ is the region of $u_{1}$ corresponding to $u \in \mathcal{U}$ and $u_{1 e}$ is the steady-state equilibrium point corresponding to $u=u_{e}$. An easy and often effective choice of system $D$ is the real diagonal constant matrix $D_{l}$ obtained in Step 3. Solve the steady-state equation and find a unique function $x=h\left(u_{1}\right)$ from the equation $0=f\left(x, u_{1}\right)$. Check whether $x=h\left(u_{1}\right) \in \mathrm{C}^{2}$ for any $u_{1} \in \mathcal{U}_{1}$. If not, DIC is not conclusive. Otherwise, proceed to the next step.

Step 5. Redefine the input $\Delta u$ as $u_{1}-u_{1 e}$ and output $\Delta y$ as $y-y_{e}$ such that the steady-state input output function $\Delta y=g(h(\Delta u), \Delta u)$ is unbiased in the sense that $0=g(h(0), 0)$. Then, check Condition (iii) of Theorem 3 for positiveness of the inner product $\Delta y^{T} \Delta u=\Delta u^{T} g(h(\Delta u), \Delta u)$ numerically in the region of interest. The condition (to ensure LES) of $\Delta u^{T} g(h(\Delta u), \Delta u) \geq \rho|\Delta u|^{2}$ (for some scalar $\rho>0$ ) for $\Delta u$ in a neighborhood of $\Delta u=0$ is guaranteed by the use of $D_{l}$ as system $D$. If the inner product $\Delta y^{T} \Delta u$ satisfies Condition (iii) at the region of interest, then the DIC of the process $P$ is concluded.

To illustrate the above procedure, the DIC of a dual tank level control system is analyzed. As shown in Figure 3, water flows into two tanks and is controlled by Pump 1 and Pump 2 to produce flow rates $f_{1}$ and $f_{2}$. The outlet flow rates of the two tanks are denoted $f_{o 1}$ and $f_{o 2}$ respectively. The liquid levels in tank 1 and tank 2 are $h_{1}$ and $h_{2}$. Assume $h_{1}>h_{2}$ and the flow rate from tank 1 to tank 2 is $f_{12}$.

The liquid levels in tank 1 and 2 can be described by the equation

$$
\left\{\begin{aligned}
\frac{d h_{1}}{d t} & =\frac{1}{A}\left(-f_{12}-f_{o 1}+f_{1}\right)=\frac{1}{A}\left(-k_{2} \sqrt{h_{1}-h_{2}}-k_{1} \sqrt{h_{1}}+f_{1}\right) \\
\frac{d h_{2}}{d t} & =\frac{1}{A}\left(f_{12}-f_{o 2}+f_{2}\right)=\frac{1}{A}\left(k_{2} \sqrt{h_{1}-h_{2}}-k_{1} \sqrt{h_{2}}+f_{2}\right) .
\end{aligned}\right.
$$

where $A=1 \mathrm{~m}^{2}, k_{1}=0.26 \mathrm{~m}^{-\frac{1}{2}} / \mathrm{min}, k_{2}=0.13 \mathrm{~m}^{-\frac{1}{2}} / \mathrm{min}$. If we select the flow rates $f_{1}$ and $f_{2}$ as control inputs and the heights of the liquid level $h_{1}$ and $h_{2}$ as control outputs, then the process model (5) can be 


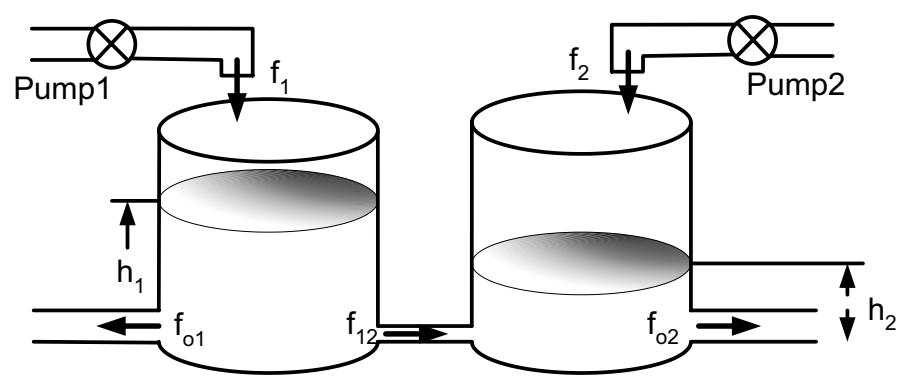

Figure 3: Schematic diagram of a dual tank level control process

written as

$$
\begin{aligned}
& \left\{\begin{array}{l}
\dot{x}_{1}=\frac{1}{A}\left(-k_{2} \sqrt{x_{1}-x_{2}}-k_{1} \sqrt{x_{1}}+u_{1}\right) \\
\dot{x}_{2}=\frac{1}{A}\left(k_{2} \sqrt{x_{1}-x_{2}}-k_{1} \sqrt{x_{2}}+u_{2}\right) .
\end{array}\right. \\
& \left\{\begin{array}{l}
y_{1}=x_{1} \\
y_{2}=x_{2}
\end{array}\right.
\end{aligned}
$$

Locally Exponential Stability of process model(6) can be confirmed by proving the stability of its linearized model around the equilibrium. The proof of Globally Asymptotical Stability of model (6) in the region of interest $\left(h_{1}>0, h_{2}>\right.$, and $\left.h_{1}-h_{2}>0\right)$ is shown in Appendix B. The DIC property of this process around an equilibrium point $x_{e}=\left[h_{1 e}, h_{2 e}\right]^{T}=[8.8,5.8]^{T} \mathrm{~m}$ is analyzed as follows. Firstly, linearization around this equilibrium is performed. It is verified that the condition (4) can be satisfied by $D=I$. Therefore, the linearized system is DIC, and satisfies all the necessary conditions for local DIC. The conditions of Theorem 3 for the nonlinear model (6) and (7) are analyzed using $D=I$.

By redefining the state $\tilde{x}=x-x_{e}=\left[x_{1}-8.8, x_{2}-5.8\right]^{T} \mathrm{~m}$, and also the input $\Delta u=u_{1}-u_{e}=\left[u_{11}-\right.$ $\left.1, u_{12}-0.4\right]^{T} m^{3} / \mathrm{min}$ and output as $\Delta y=y-y_{e}=\left[y_{1}-8.8, y_{2}-5.8\right]^{T} m^{3} / \mathrm{min}$, the steady-state input output mapping can be found as follows:

$$
\left\{\begin{aligned}
& \Delta y_{1}=\left(25 \sqrt{5\left(1+\Delta u_{1}\right)^{2}+2\left(1+\Delta u_{1}\right)\left(2 / 5+\Delta u_{2}\right)-3\left(2 / 5+\Delta u_{2}\right)^{2}}\right. \\
&\left.-135-125 \Delta u_{1}-25 \Delta u_{2}\right)^{2} / 676-8.8 \\
& \Delta y_{2}=\left(25 \sqrt{5\left(1+\Delta u_{1}\right)^{2}+2\left(1+\Delta u_{1}\right)\left(2 / 5+\Delta u_{2}\right)-3\left(2 / 5+\Delta u_{2}\right)^{2}}\right. \\
&\left.+5-25 \Delta u_{1}+75 \Delta u_{2}\right)^{2} / 676-5.8
\end{aligned}\right.
$$

Condition iii) in Theorem 3 is reduced to $\Delta y^{T} \Delta u>0$. Because function $\Delta y^{T} \Delta u$ is continuous, it can be verified numerically using discrete points in the region of interest. A three-dimensional plot is given in Figure 4 from which it can be seen that the DIC conditions in Theorem 3 are satisfied in the region $\Delta u_{1} \in[-0.4,0.4] \mathrm{m}^{3} / \mathrm{min}\left(f_{1} \in[0.6,1.4] \mathrm{m}^{3} / \mathrm{min}\right)$ and $\Delta u_{2} \in[-0.2,0.2] \mathrm{m}^{3} / \mathrm{min}\left(f_{2} \in[0.2,0.6] \mathrm{m}^{3} / \mathrm{min}\right)$. Therefore, this nonlinear process is DIC when the control input is in this region.

\section{Conclusion}

This paper gives an extension of the concept of Decentralized Integral Controllability to nonlinear processes, and provides a sufficient DIC condition for the analysis of nonlinear process. This condition only requires steady-state models, which are often easy to obtain. It is also shown that this sufficient condition can be regarded as a generalization of the existing linear DIC condition (Bao et al. 2002). Furthermore, a 


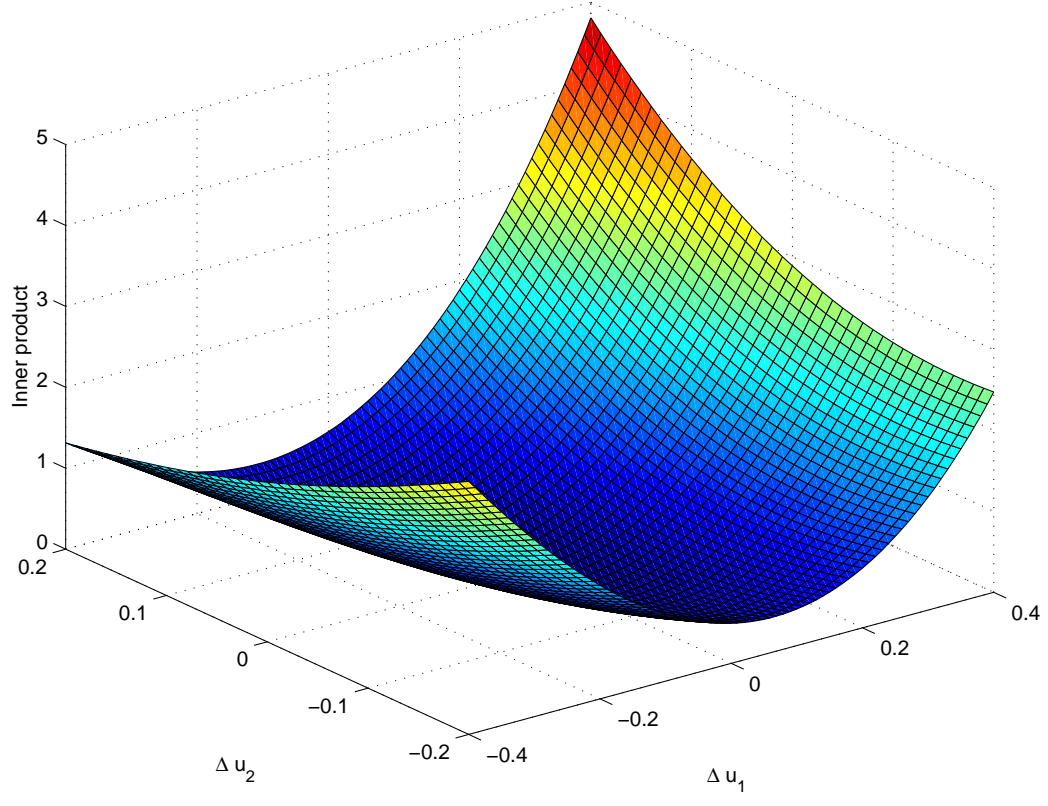

Figure 4: Values of $\Delta y^{T} \Delta u$.

numerical method for verification of the proposed condition is proposed to assist the analysis of nonlinear DIC condition. The DIC analysis of the dual tank level control system is performed to illustrate the DIC condition and numerical method.

\section{Acknowledgments}

The authors gratefully acknowledge the financial support of the Australian Research Council (Grant A00104473).

\section{References}

Bao, J., McLellan, P. \& Forbes, J. (2002), 'A passivity-based analysis for decentalised integral controllability', Automatica 30, 243-247.

Campo, P. \& Morari, M. (1994), 'Achievable closed-loop properties of systems under decentralised control: conditions involving the steady state gain', IEEE Trans. Automatic Control 39(5), 932-943.

Kokotovic, P., Khalil, H. \& Reilly, J. (1986), Singular Perturbation Methods in Control: Analysis and Design, Academic Press Inc, U.S.A.

Le, D., Nwokah, O. \& Frazho, A. (1991), 'Multivariable decentralized integral controllability', International Journal of Control 54(2), 481-496.

Lee, J. \& Edgar, T. (2002), 'Conditions for decentralized integral controllability', Journal of Process Control 12(7), 797-805.

Luyben, W., Tyrus, B. \& Luyben, M. (1999), Plantwide Process Control, McGraw-Hill, N.Y. 
Morari, M. \& Zafiriou, E. (1989), Robust Process Control, Prentice Hall, Englewood Cliffs, NJ.

Nikolaou, M. \& Misra, P. (2003), 'Linear control of nonlinear processes:recent developments and future directions', Computers and Chemical Engineering 27, 1043-1059.

Nwokah, O., Frazho, A. \& Le, D. (1993), 'A note on decentralized integral controllability', International Journal of Control 57(2), 485-494.

Sepulchre, R., Jankovic, M. \& Kokotovic, P. (1996), Constructive Nonlinear Control, Springer Verlag, New York.

Skogestad, S. \& Morari, M. (1988), 'Variable selection for decentralized control', AIChE Annual Meeting, Washington, DC p. Paper 128c.

Vinson, D. \& Georgakis, C. (1999), 'A new measure of process output controllability', J. Proc. Cont. 10, 185194.

Yu, C. \& Fan, M. (1990), 'Decentralized integral controllability and d-stability', Chemical Engineering Science 45(11), 3299-3309.

\section{A Proof of Theorem 3.}

Proof The proof of this Theorem is based on singular perturbation theory (Kokotovic et al. 1986, Sepulchre et al. 1996).

Consider the system of Figure 2 described by equations (2) and (3). The input signal $r$ is set to zero so that the Lyapunov stability of the unforced closed loop $\left(P,-C_{l}\right)$ can be analyzed. The state equation for the closed loop $\left(P,-C_{l}\right)$ can be expressed as:

$$
\left(P,-C_{l}\right):\left\{\begin{array}{l}
\dot{x}=f(x, \xi) \\
\dot{\xi}=-\eta K_{g \varepsilon}^{\prime} g(x, \xi) .
\end{array}\right.
$$

Equation (9) can be transformed into a standard singular perturbation form (Kokotovic et al. 1986): Let $\tau=\eta\left(t-t_{0}\right)$, so that $\tau=0$ at $t=t_{0}$. As $\frac{d \tau}{d t}=\eta$, we have:

$$
\left(P,-C_{l}\right):\left\{\begin{aligned}
\eta \frac{d}{d \tau} x & =f(x, \xi) \\
\frac{d}{d \tau} \xi & =-K_{g \varepsilon}^{\prime} g(x, \xi) .
\end{aligned}\right.
$$

In order to be consistent with standard singular perturbation notation, we will for the moment use the notation $\dot{x}$ to denote the derivative on the slow time scale $\tau$ when we analyze singular perturbation models. For the standard singular perturbation model (10), the following conclusions can be drawn based on Condition i), ii) and iii) respectively:

(i) The equation $0=f(x, \xi)$ obtained by setting $\eta=0$ in equation (10) implicitly defines a unique $C^{2}$ function $x=h(\xi)$.

(ii) For any fixed $\xi \in \mathcal{R}^{m}$, the equilibrium $x_{e}=h(\xi)$ of the subsystem $\dot{x}=f(x, \xi)$ is Globally Asymptotically Stable (GAS) (Sepulchre et al. 1996) and Locally Exponentially Stable (LES).

(iii) The equilibrium $\xi=0$ of the reduced model (slow time scale) $\dot{\xi}=-K_{g \varepsilon}^{\prime} g(h(\xi), \xi)$ is GAS and LES (See 
Definition 2 ).

Both conclusions (i) and (ii) are obvious. Only Conclusion (iii), the stability(GAS and LES) of "slow time scale", needs to be proved.

Assume $K_{g \varepsilon}^{\prime}>0$ at first. Consider $V(\xi)=\frac{1}{2} \xi^{T} K_{g \varepsilon}^{\prime-1} \xi$ as a Lyapunov function candidate for the "slow time scale". It can be seen that

$$
\dot{V}(\xi)=-\frac{1}{2}\left[\xi^{T} K_{g \varepsilon}^{\prime-1} K_{g \varepsilon}^{\prime} g(h(\xi), \xi)+g^{T}(h(\xi), \xi) K_{g \varepsilon}^{\prime} K_{g \varepsilon}^{\prime-1} \xi\right]=-g^{T}(h(\xi), \xi) \xi=-\xi^{T} g(h(\xi), \xi)
$$

This will satisfy the requirements for GAS and LES given that $u_{1}^{T} g\left(h\left(u_{1}\right), u_{1}\right)>0\left(\right.$ when $\left.u_{1} \neq 0\right)$ and $u_{1}^{T} g\left(h\left(u_{1}\right), u_{1}\right) \geq \rho\left|u_{1}\right|^{2}$ (for some scalar $\rho>0$ ) for $u_{1}$ in a neighborhood of $u_{1}=0$. It should be noted that the GAS and LES of the "slow time scale" is guaranteed for all $K_{g \varepsilon}^{\prime}=\operatorname{diag}\left\{k_{i}^{\prime} \varepsilon_{i}\right\}=\operatorname{diag}\left\{\frac{k_{i} \varepsilon_{i}}{\eta}\right\}, 0<\varepsilon_{i} \leq$ $1, i=1, \cdots, m$.

Now, consider the case that $K_{g \varepsilon}^{\prime} \geq 0$. That is, some diagonal elements of $K_{g \varepsilon}^{\prime}$ are zero and the corresponding controller loops are removed. Without loss of generality, assume $\varepsilon_{j}=\varepsilon_{l}=0$, where $1 \leq j<l \leq m$. Denote $\bar{\xi}=\left[\xi_{1}, \cdots, \xi_{j-1}, \xi_{j+1}, \cdots, \xi_{l-1}, \xi_{l+1}, \cdots \xi_{m}\right]^{T}, \tilde{\xi}=\left[\xi_{1}, \cdots, \xi_{j-1}, 0, \xi_{j+1}, \cdots, \xi_{l-1}, 0, \xi_{l+1}, \cdots \xi_{m}\right]^{T}$, $\bar{g}(h(\tilde{\xi}), \tilde{\xi})=\left[g_{1}(h(\tilde{\xi}), \tilde{\xi}), \cdots, g_{j-1}(h(\tilde{\xi}), \tilde{\xi}), g_{j+1}(h(\tilde{\xi}), \tilde{\xi}), \cdots, g_{l-1}(h(\tilde{\xi}), \tilde{\xi}), g_{l+1}(h(\tilde{\xi}), \tilde{\xi}), \cdots\right.$, $\left.g_{m}(h(\tilde{\xi}), \tilde{\xi})\right]^{T}$ and $\bar{K}_{g \varepsilon}^{\prime}=\operatorname{diag}\left\{k_{1}^{\prime} \varepsilon_{1}, \cdots, k_{j-1}^{\prime} \varepsilon_{j-1}, k_{j+1}^{\prime} \varepsilon_{j+1}, \cdots, k_{l-1}^{\prime} \varepsilon_{l-1}, k_{l+1}^{\prime} \varepsilon_{l+1}, \cdots, k_{m}^{\prime} \varepsilon_{m}\right\}$. Then, the model of "slow time scale" can be rewritten as

$$
\dot{\bar{\xi}}=-\bar{K}_{g \varepsilon}^{\prime} \bar{g}(h(\tilde{\xi}), \tilde{\xi})
$$

Choose $\bar{V}(\bar{\xi})=\frac{1}{2} \bar{\xi}^{T} \bar{K}_{g \varepsilon}^{\prime-1} \bar{\xi}$ as a Lyapunov function candidate for the "slow time scale" model (12), then

$$
\dot{\bar{V}}(\bar{\xi})=-\bar{\xi}^{T} \bar{g}(h(\tilde{\xi}), \tilde{\xi})
$$

The condition $u_{1}^{T} g\left(h\left(u_{1}\right), u_{1}\right)>0\left(\right.$ when $u_{1} \neq 0$ ) implies that $\bar{\xi}^{T} \bar{g}(h(\tilde{\xi}), \tilde{\xi})>0$ (when $\bar{\xi} \neq 0$ ), and $u_{1}^{T}$ $g\left(h\left(u_{1}\right), u_{1}\right) \geq \rho\left|u_{1}\right|^{2}$ (for some scalar $\rho>0$ ) for $u_{1}$ in a neighborhood of $u_{1}=0 \operatorname{implies}$ that $\bar{\xi}^{T} \bar{g}(h(\tilde{\xi}), \tilde{\xi}) \geq$ $\rho^{\prime}|\bar{\xi}|^{2}$ (for some scalar $\rho^{\prime}>0$ ) for $\bar{\xi}$ in a neighborhood of $\bar{\xi}=0$. Therefore, GAS and LES of the "slow time scale" model (12) can be proved.

Then, the conclusion of this theorem follows from Theorem 3.18 in (Sepulchre et al. 1996).

\section{B Proof of the Asymptotically Stability of process model (6).}

Proof By redefining the state $\tilde{x}$ as $x-x_{e}$, and also the input $\tilde{u}$ as $u-u_{e}$, the process (6) can be translated as follows:

$$
\left\{\begin{array}{l}
\dot{\tilde{x}}_{1}=-k_{2} \sqrt{\tilde{x}_{1}-\tilde{x}_{2}+x_{1 e}-x_{2 e}}-k_{1} \sqrt{\tilde{x}_{1}+x_{1 e}}+\tilde{u}_{1}+u_{1 e} \\
\dot{\tilde{x}}_{2}=k_{2} \sqrt{\tilde{x}_{1}-\tilde{x}_{2}+x_{1 e}-x_{2 e}}-k_{1} \sqrt{\tilde{x}_{2}+x_{2 e}}+\tilde{u}_{2}+u_{2 e} .
\end{array}\right.
$$

If we set $u_{1 e}=k_{2} \sqrt{x_{1 e}-x_{2 e}}+k_{1} \sqrt{x_{1 e}}$ and $u_{2 e}=-k_{2} \sqrt{x_{1 e}-x_{2 e}}+k_{1} \sqrt{x_{2 e}}$, then $x_{e}\left(x_{1 e}>x_{2 e}\right)$ is the equilibrium of model (14). A Lyapunov function for the unforced model (14) is selected as $V\left(\tilde{x^{2}}\right)=\frac{1}{2}\left({\tilde{x_{1}}}^{2}+\right.$ 
${\tilde{x_{2}}}^{2}$. The new input $\tilde{u}$ is set to be zero when the Lyapunov stability of the model (14) is analyzed. Then,

$$
\begin{aligned}
\dot{V}(\tilde{x})= & k_{2}\left(\tilde{x}_{1}-\tilde{x}_{2}\right)\left(\sqrt{x_{1 e}-x_{2 e}}-\sqrt{\tilde{x}_{1}-\tilde{x}_{2}+x_{1 e}-x_{2 e}}\right) \\
& +k_{1} \tilde{x}_{1}\left(\sqrt{x_{1 e}}-\sqrt{\tilde{x}_{1}+x_{1 e}}\right)+k_{1} \tilde{x}_{2}\left(\sqrt{x_{2 e}}-\sqrt{\tilde{x}_{2}+x_{2 e}}\right) \\
= & -\frac{k_{2}\left(\tilde{x}_{1}-\tilde{x}_{2}\right)^{2}}{\sqrt{x_{1 e}-x_{2 e}+\sqrt{\tilde{x}_{1}-\tilde{x}_{2}+x_{1 e}-x_{2 e}}}-\frac{k_{1} \tilde{x}_{1}{ }^{2}}{\sqrt{x_{1 e}}+\sqrt{\tilde{x}_{1}+x_{1 e}}}} \\
& -\frac{k_{1} \tilde{x}_{2}^{2}}{\sqrt{x_{2 e}}+\sqrt{\tilde{x}_{2}+x_{2 e}}} .
\end{aligned}
$$

From equation (15), it can be seen that $\dot{V}(\tilde{x})=0$ if and only if $\tilde{x}=0$, and $\dot{V}(\tilde{x}) \leq 0$ when $\tilde{x}_{i}+x_{i e} \geq 0$ $\left(x_{i} \geq 0\right)$ for $i=1,2$, and $\tilde{x}_{1}-\tilde{x}_{2}+x_{1 e}-x_{2 e} \geq 0\left(x_{1} \geq x_{2}\right)$.

Thus, model (6) is GAS around an equilibrium $x_{e}$ in the region of interest. 